\title{
Sensor Nodes and System Proposal for Security and Environmental Data Collection in Developing Countries
}

\author{
Pierre Tsafack $^{1}$, Valery Nkemeni ${ }^{1}$, Bruno Allard ${ }^{2}$ \\ ${ }^{1}$ Department of Electrical and Electronics Engineering, University of Buea, Buea, Cameroon \\ ${ }^{2}$ Department of Electrical Engineering, Institut National des Sciences Appliquées de Lyon, Lyon, France
}

Email address:

pierro_tsafack@yahoo.com (P. Tsafack),nkemvale@gmail.com (V. Nkemeni), bruno.allard@insa-lyon.fr (B. Allard)

\section{To cite this article:}

Pierre Tsafack, Valery Nkemeni, Bruno Allard. Sensor Nodes and System Proposal for Security and Environmental Data Collection in Developing Countries. Industrial Engineering. Vol. 4, No. 1, 2020, pp. 7-13. doi: 10.11648/j.ie.20200401.12

Received: May 18, 2020; Accepted: June 10, 2020; Published: August 13, 2020

\begin{abstract}
This paper consists of RF-based sensor nodes design for survey of critical areas and distance collection of useful environmental data such as pollutants, pluviometry, temperature and humidity. Given poor or nonexistent accesses in areas under investigation, adequate RF equipped sensor nodes are posted at indicated locations for collecting data to be processed by a microcontroller used as a control and processing unit. The processing unit also processes requests from users and sends feedbacks by SMS; those requests and related feedbacks are transmitted through a GSM module. Data from sensors are also processed and corresponding SMS alerts or calls are automatically sent when set parameters are detected. In addition to the use of GSM and microcontroller-based processing unit as in few systems, the proposed system here used RF-based sensor nodes for local area data sensing. That allows real time investigation of given locations. The prototype was successfully developed and tested with specific COTS components such as RF TX/RX $433 \mathrm{MHz}$ for local area communication, PLL4046 for data formatting to digital, GSM SIM900 for long distance communication, and PIC16F877 microcontroller for data processing.
\end{abstract}

Keywords: COTS Components, GSM, Microcontroller, Sensors, Environmental Data, Security, Developing Countries

\section{Introduction}

Security and control systems have moved from wired to wireless methods over the years; some of which use ZigBee, LoRa, Bluetooth, GSM and Wi-Fi technology amongst others. GSM has proven to be one of the most efficient method for communication/survey systems in developing countries thank to the existing well deployed network all through areas and to the SMS service it provides. It has therefore been one of the most used technology for security and control systems.

In Cameroonian local communities like in many other developing countries, people and properties are in high need of security services for safety purposes due to the everincreasing security threats faced on a daily basis by citizens. Given the on-going fast climatic changes, there is also a great need for a constant survey of environment through an efficient real time collection of related data using adequate sensor nodes.

Hence, this work has as a major objective to investigate communication modules and technologies for a design of a long range, cost effective, efficient and user-friendly security and environmental data monitoring system. RF-based sensor nodes are therefore used for accessible and inaccessible locations. This proposed system also uses the GSM module for long distance and two-way communication between the user and the system; this option allows data collection from the system on request.

To cover the above mentioned objectives, this work is organized as follow: section two gives a review of the various technologies used for communication, security and control; a comparison of these technologies and some related works which use GSM for security and control; in section three, the adopted methodology as well as a system proposal and analysis are presented. Implementation, results and discussion are presented in section four. We end with a conclusion and future scope in section five. 


\section{Review of Technologies, Communication, Control and Security Systems}

Although wired systems are still in use because of some of their advantages over wireless Local Area Network (LAN), wireless security and control systems are becoming more and more popular. This is due to their flexibility to install because of no cabling, easy system upgrading, automation capability and most importantly remote accessibility. The most used wireless technologies for control and security include Z-wave,
ZigBee, Wi-Fi, Bluetooth, LoRa and GSM.

\subsection{Comparison of Wireless Technologies}

The Table 1 below is a comparison table of the abovementioned wireless communication technologies. Based on that, it can be observed and concluded as to why GSM is chosen for this work. As a matter of fact, GSM comparatively offers a wider range over the other technologies; it is locally available as the cellular network has already been deployed locally; that helps to reduce the implementation cost; it is also compatible with most if not all devices used for security and control and it is user-friendly.

Table 1. Comparison of wireless communication technologies [1-3, 12, 13].

\begin{tabular}{|c|c|c|c|c|c|c|}
\hline FEATURE & GSM & LORA & WI-FI & BLUETOOTH & ZIGBEE & Z-WAVE \\
\hline Frequency & $\begin{array}{l}850 \mathrm{MHz} / 1.9 \mathrm{G} \\
\mathrm{Hz}\end{array}$ & $\begin{array}{l}868 \mathrm{MHz} \\
915 \mathrm{MHz}\end{array}$ & $2.4 \mathrm{GHz}$ & $2.4 \mathrm{GHz}$ & $\begin{array}{l}2.4 \mathrm{GHz} \\
868 \mathrm{MHz} \\
915 \mathrm{MHz}\end{array}$ & $\begin{array}{l}868 \mathrm{MHz} \\
908 \mathrm{MHz}\end{array}$ \\
\hline Type & WAN & LPWAN & LAN & PAN & PAN & PAN \\
\hline Power & Very High & Low & High & Low & Very Low & Very Low \\
\hline Battery Life & Hours & Years & Hours & Days & Months and years & Months and years \\
\hline Mesh & No & No & 30 & 7 & 65,000 & 232 \\
\hline Bandwidth & $135 \mathrm{Kbps}$ & $27 \mathrm{Kbps}$ & $2-100 \mathrm{Mbps}$ & $1 \mathrm{Mbps}$ & $20-250 \mathrm{Kbps}$ & $100 \mathrm{Kbps}$ \\
\hline Range (m) & 35,000 & 15,000 & $1-100+$ & $1-100$ & $1-30,100$ & 150 \\
\hline Cost & Costly & Less costly & Cheap & Cheap & Cheap & Cheap \\
\hline Local availability of module & Yes & No & Yes & Yes & No & Yes \\
\hline Compatibility with mobile /laptop & Yes & No & Yes & Yes & No & Yes \\
\hline
\end{tabular}

\subsection{Related Works}

This section covers related literature on GSM-based remote access, control and security in different aspects.

\subsubsection{GSM Security and Monitoring Systems: Use of PC as Processing Unit}

GSM security and monitoring systems raised up since the early 2000s. The earlier system implementation made use of PCs for control and sometimes a combination of GSM and other technologies for communication. Several security systems based on sensor network were therefore proposed [4, $5,16,17]$. For example, in 2009, a system based on GSMSMS technology was designed for controlling home appliances remotely [4]. Whereby a client mobile phone could communicate with server mobile phone via an existing GSM network with messages delivered via SMS (short messaging service). This system made use of both the Bluetooth technology for remote access control within the home and the GSM-SMS technology to access the appliances remotely from a long distance outside the home. The server mobile connected to the server PC via Bluetooth and the PC connected to appliances via a circuit network and on the other hand, the server mobile connected to the client mobile via GSM network, ensured this remote access when the owner is home and far from home [4]. Figure 1 is the architecture of a such system. One major drawback of this design was the use of computer to control the system which is very expensive. Later on, microcontrollers were used to reduce cost.

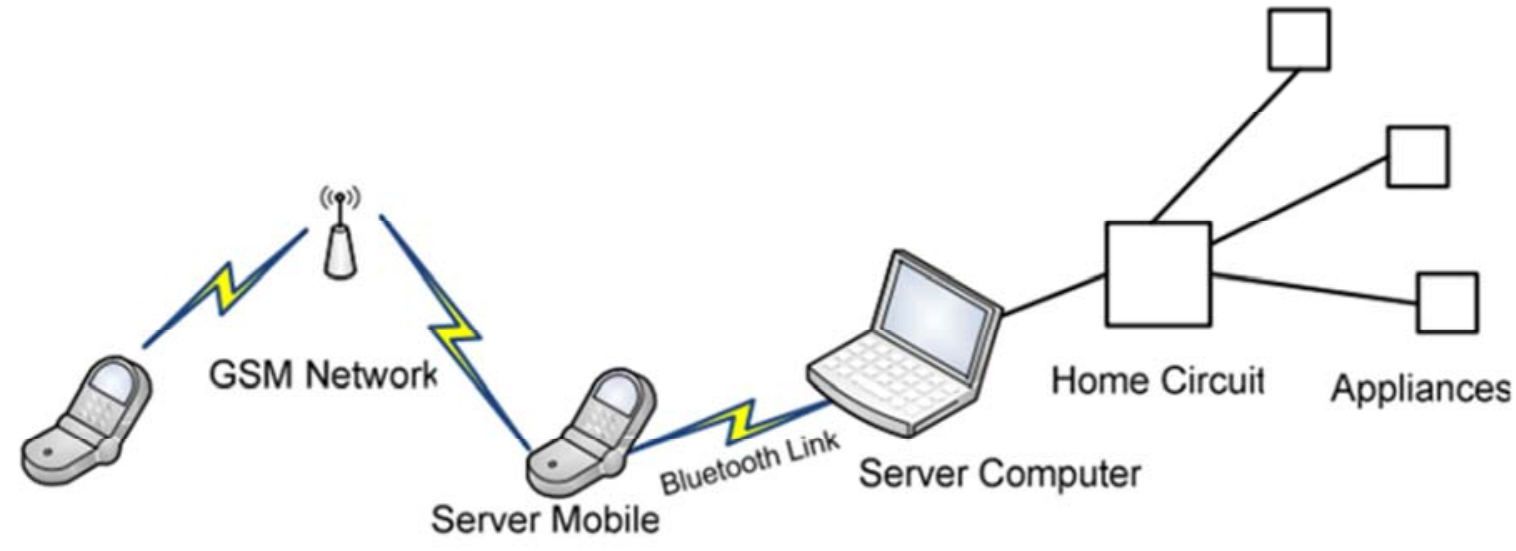

Figure 1. Architecture, GSM-SMS technology for controlling home appliances remotely [4]. 


\subsubsection{GSM Security and Monitoring Systems: Use of Microcontroller as Processing Unit}

By March 2012, computer control was almost already eliminated with the use of microcontrollers in order to reduce cost and for better effectiveness [5, 6, 14-16]. For instance, a GSM was proposed by Sadeque Rezafor Khan et al. for low cost security for homes [5]. The project was aimed at developing the security of home against intruders and fire. It made use of the PIC microcontroller, GSM module and several sensors such as heat sensor, obstacle sensor, smoke sensor, sound sensor and touch sensor. The house owner, the police or even the fire brigade was notified immediately in the event of an unauthorized incident. Figure 2 is an illustration of a such system.

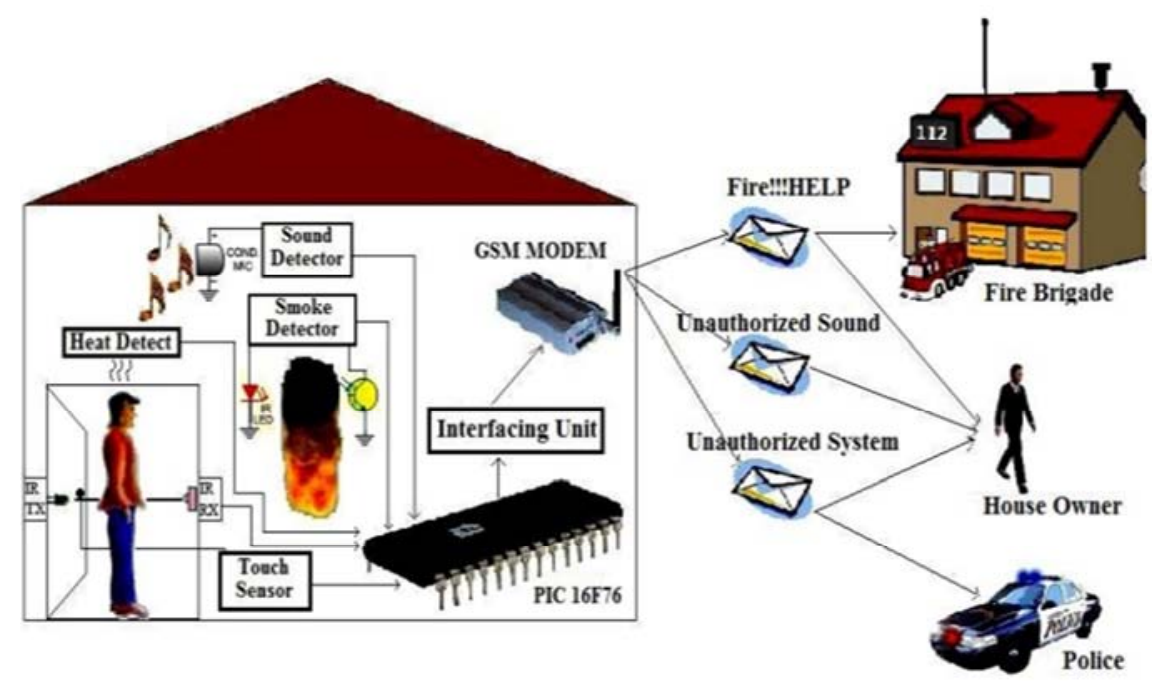

Figure 2. Illustration of Low-Cost Home Security System using GSM and PIC microcontroller [5].

In December 2017, Chandrakant Bhadranna, proposed a design of a security system for a computer lab [6]. PIR sensors were used in this case to detect the presence of humans for theft prevention. He used ATMEL family microcontroller to control the system and IP camera to capture images and videos; a GSM module was included for communication between the system and the registered numbers. The video of the intruder could be watched in live on the owner's mobile phone and this was made possible with the use of the IP cameras at the time of motion detection.

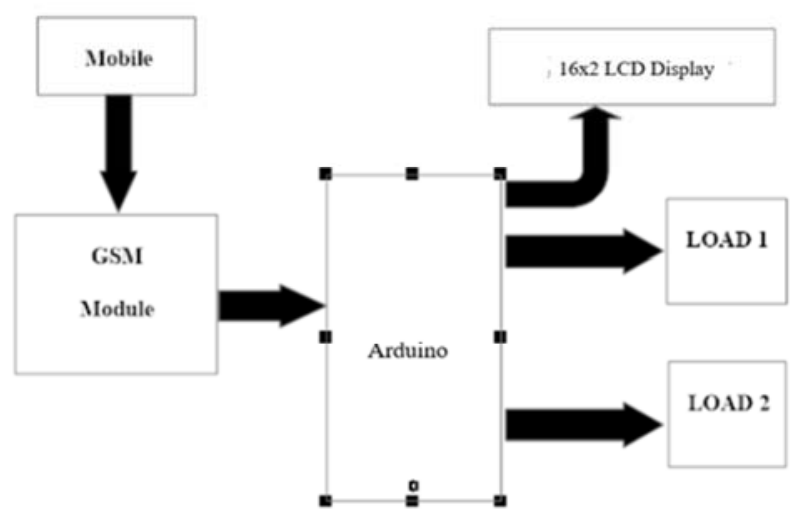

Figure 3. Block Diagram of GSM based home automation system [7].

Another system titled GSM based home automation system was proposed by Yusuf Sharif et al. [7]. The system was designed to control electrical devices throughout the house with ease of installing, ease of use and cost-effective design and implementation. It consisted of an Arduino-Uno board, a GSM Module, a GSM based phone, power sockets and home appliances. The GSM SIM provided the communication media between the home owner and the system by means of SMS. The SMS consisted of commands in written form to be executed. The format of the message was predefined. The SMS message was sent to the GSM SIM via the GSM network as a text message with a definite predefined format. Once the GSM SIM received the message, the commands sent could be extracted and executed by the microcontroller and turn the appliances ON/OFF accordingly via the switching module. Figure 3 is a block diagram of a such system. Similar to the above system, Sarkar et al. also proposed A Smart Control System of Home Appliances Using SMS [8]. The mobile unit was used as a transmitting section from which the subscriber sends text messages that contain commands and instructions.

In the same framework, Subhankar Chattoraj proposed a Smart Home Automation based on different sensors and Arduino as the master controller [9]. The proposed model consisted of two main hardware components: The Arduino Uno microcontroller board which is flexible, inexpensive, offers a variety of digital and analog inputs, serial interface and PWM outputs; a PC home server which hosts the Matlab-GUI (Graphical User Interface) platform management. Arduino Uno controlled the home appliances and also enabled the user to access them through voice commands. The computer communicated with the Arduino Uno microcontroller board through USB data transfer cable. A number of appliances and sensors were connected to the 
communication ports of the microcontroller board and the home Appliances could be monitored and accessed remotely [9]. Figure 4 is a block diagram of a such system.

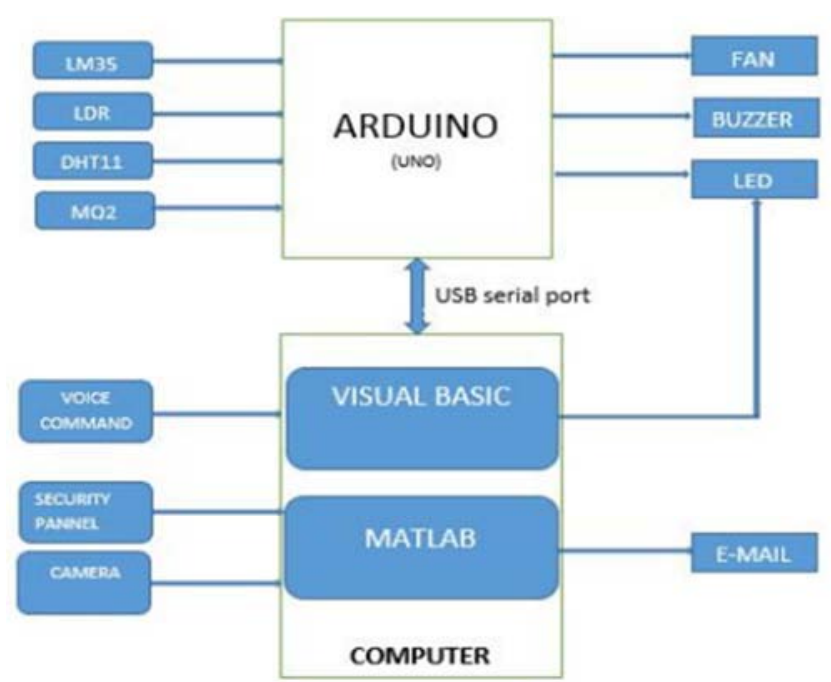

Figure 4. Block diagram of Smart Home Automation based on Arduino \& Personal Computer [9].

\section{Proposed System and Analysis}

\subsection{System Description}

The proposed system is made up of a microcontroller which plays a role of central processing unit; the RF-based sensing node for a survey of the environment and for short distance collected data transmission to the central processing unit; and then a GSM for long distance information and control. Pollutant node sensor, temperature node sensor and pluviometry node sensor are used for environmental data collection; sound and movement sensor nodes are used for security. A block diagram is presented on Figure 5. Similar to the sensor circuits of the previous sensor system implemented in [10], a PLL4046 [11] is used for TX/RX input data formatting through a FM modulation as presented in Figure 6(b). As a matter of fact, TX/RX RF modules can transmit only digital signals; we therefore proceed to a formatting of any collected data or information from original signal to a digital TX/RX input using PLL4046 based digital frequency modulation.

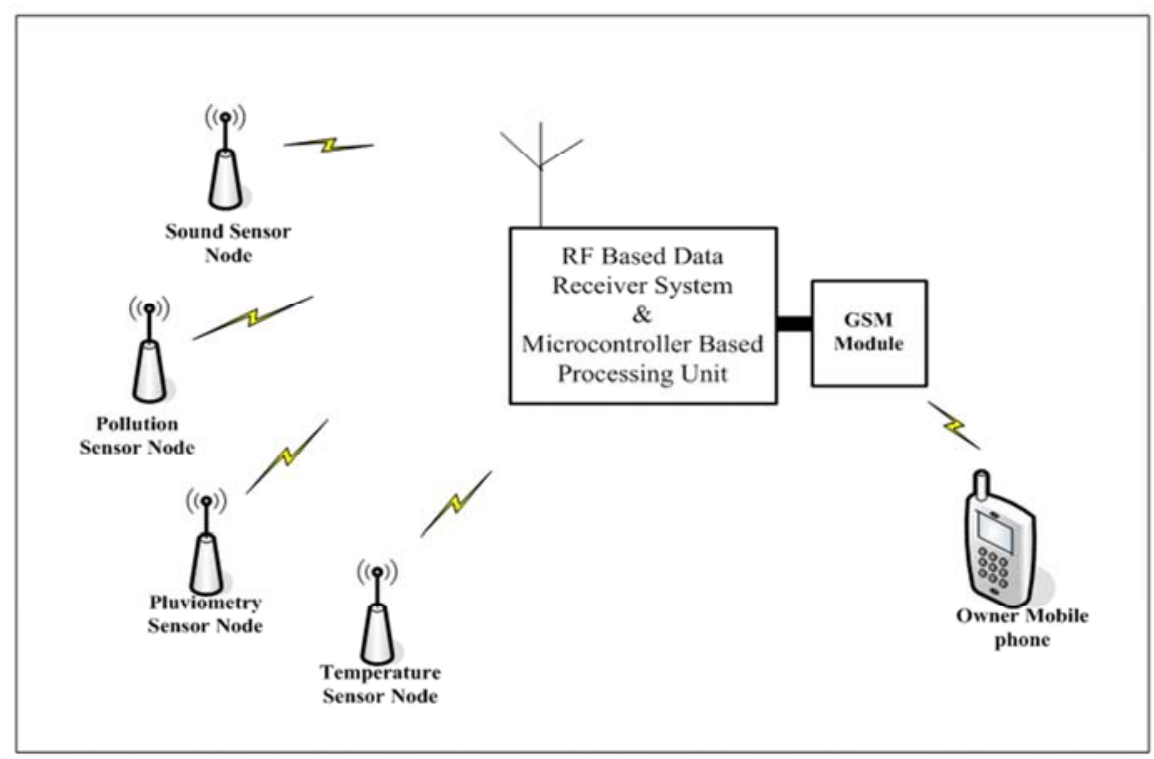

Figure 5. Block diagram of the proposed system.

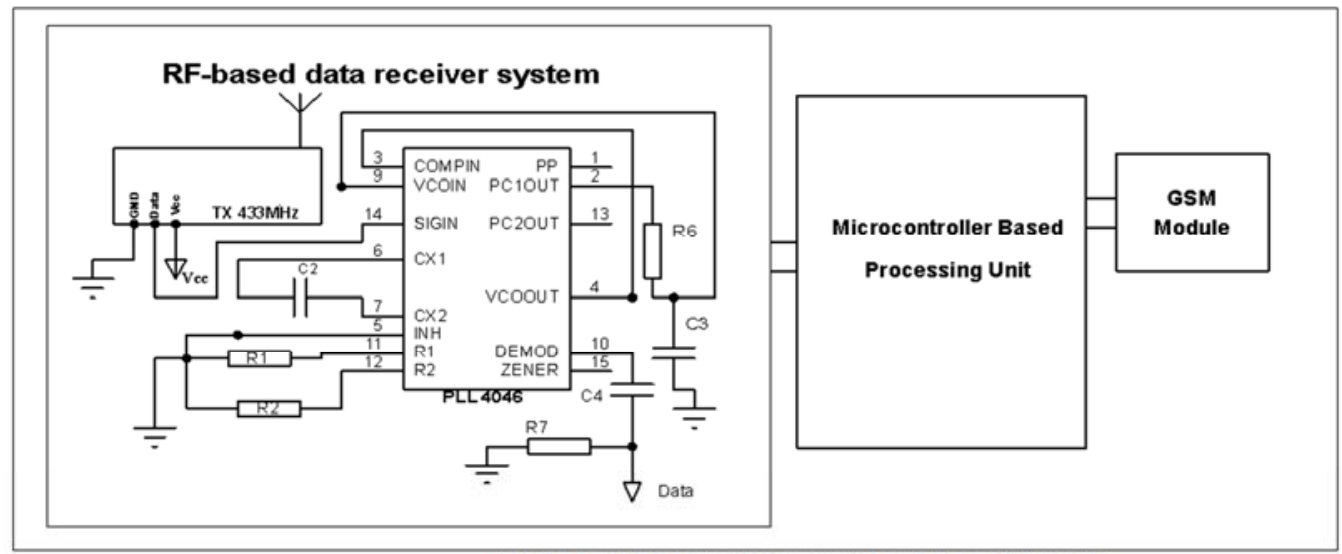

(a) RF based data receiver, control and processing node. 

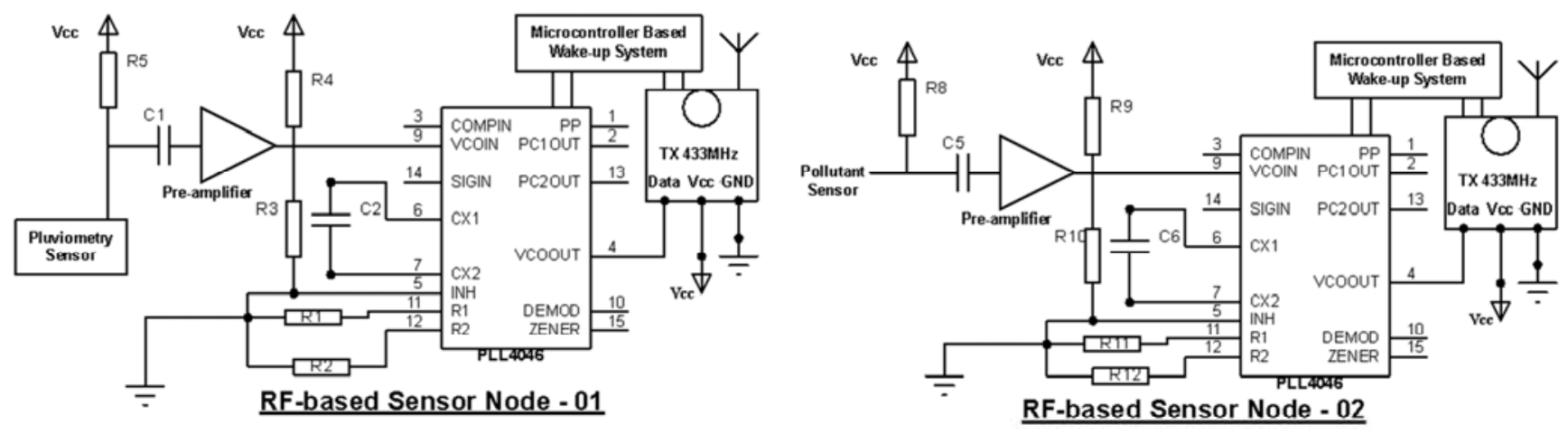

(b) Circuit samples of sensing \& RF data transmission nodes.

Figure 6. Overview of node circuits.

\subsection{Functioning Principle}

The functioning principle consists of the following activities:

Once the system is powered on, the various sensor modules and microcontroller ports are initialized; the communication tests are also performed between access point sensor nodes and the microcontroller-based processing unit. Next the GSM module is also initialized with a time delay of few second for enabling the network for communication to be established. The system runs non-stop once powered on. Within the loop, there is a check whether or not an SMS is sent to the system by the user for a control or as a request. If the system receives an SMS, it reads and performs requested instructions.

Another test within the loop is that of call. If there is a call, the GSM automatically picks the call and the user can then listen to any on-going happening around the system.

In addition to the processing of collected data/information from remote sensors, the system can take care of controlling its nearby environment using direct attached sensors.

As far as the environment follow up is concerned, we concentrated on aspects such as temperature, humidity and pluviometry; warning intervals are programmed and a SMS is sent to the user with the picked values any time the status of any of the items changes. In addition to the SMS, a call is made to the user once warning values are detected

As far as the security is concerned, if a motion is detected, to make sure it is an intruder detected, the system checks if the PIR sensor has triggered at least 5 times. If this check is positive the system places a call to the user alongside with a SMS "Intruder Detected". When the user receives the call, he/she is able to listen to the audio voice picked through the sound sensor and can take appropriate actions.

And the loop continues...

\section{Implementation, Results and Discussion}

This section covers the hardware implementation and the results obtained for this work.

\subsection{Hardware System Proposal}

The system proposal is based on COTS components such as $\mathrm{RF}$ modules $433 \mathrm{MHz}-\mathrm{TX} / \mathrm{RX}$ for short range $\mathrm{RF}$ communication of collected data/information to the central unit; SIM900A for long distance transmission of collected data and eventual long-distance request or control from users. Some sensors are also directly attached to the central unit for a control and survey of its surrounding area.

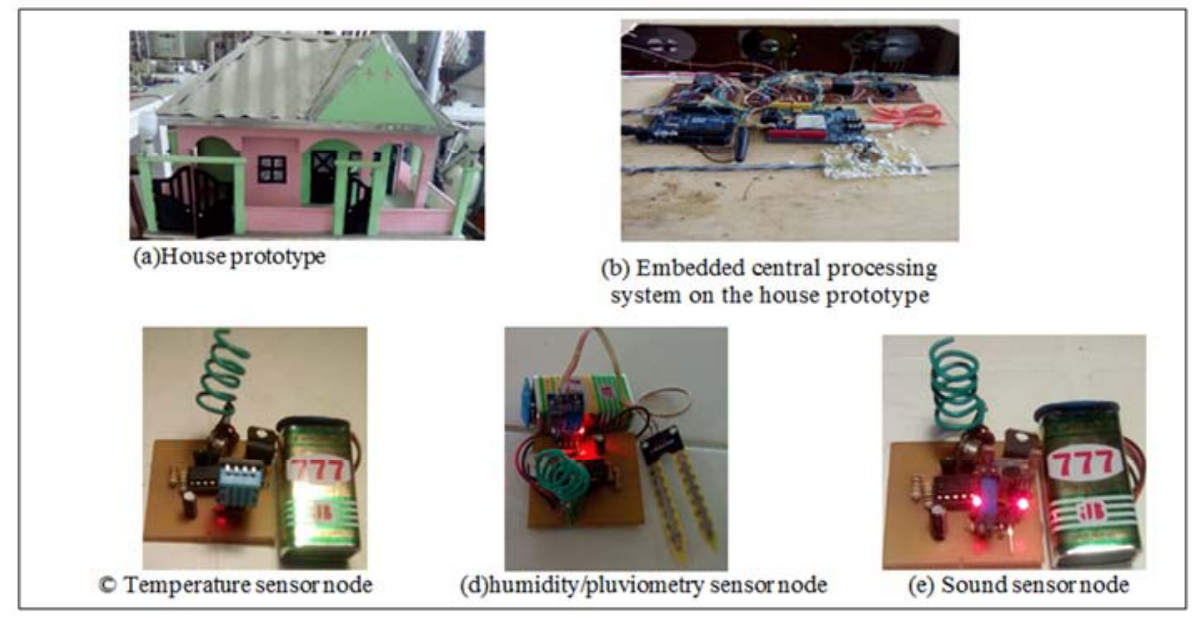

Figure 7. House and sensor nodes prototypes. 
The tests were conducted with designed remote sensor nodes such as temperature sensor node of Figure 7(c), PIR sensor and light sensor integrated on the central unit of Figure 7(b), humidity/pluviometry sensor node of Figure 7(d) and a sound sensor node of Figure 7(e). Data could be collected from local areas to the processing unit through RF modules; then made available to the user on request through SIM900A module. Necessary actions such as monitoring of security light and eventual alarm system are handled locally by the central unit and only their status is sent to the user.

A house prototype as shown on Figure 7(a) was also made for a real-life spray and testing up of remote sensors.

\subsection{Test and Results}

The results presented here are mainly to illustrate the expected functioning of the system; above sensor prototypes were used in that effect. As far as security is concerned, a remote movement sensor node monitors the area under investigation and transfer in real time its status to the central processing unit which can make a call to the user only when a desired number of motions is detected ( 5 times here) as illustrated on Figure 8(a). Also, as expected, few actions can be ordered from a distance by a user; that is illustrated here by the system receiving an "ON" message and therefore switching ON the security light bulbs as shown on Figure 8(b). The same, all changes occurring on the area are being sent in real time to the user. For instance, when darkness is detected by the LDR, security light bulbs are lit and the corresponding SMS sent to the user as presented on Figure 8(c) below. The same as indicated on Figure 8(c), temperature status is frequently sent to the central unit which keeps owners aware of synthesis after data processing; an additional warning SMS/call should be made in case of sudden increase of any indicated parameter value.

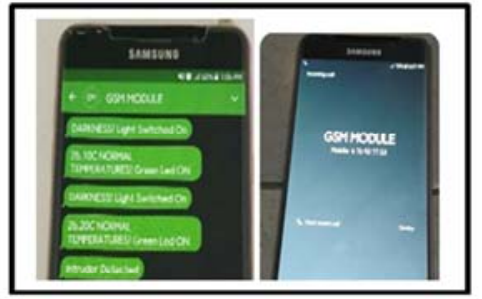

(a) SMS to user: "Intruder Detected" and GSM Call

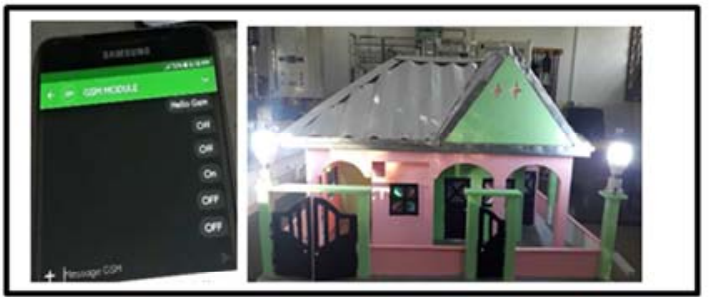

(b) SMS Control

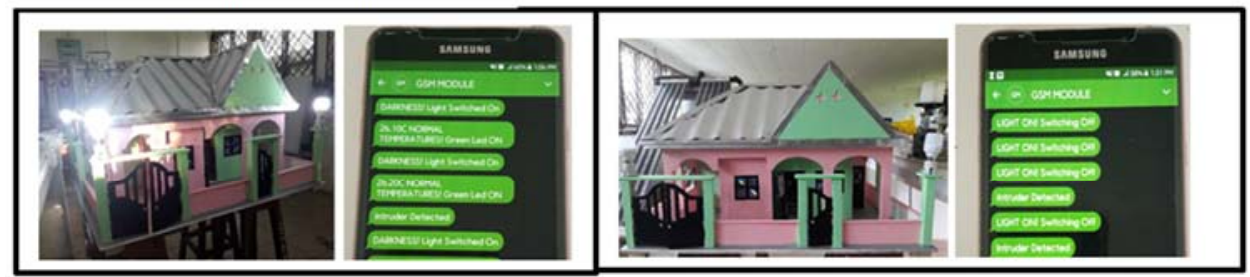

CFrequent feedback to the user: Temperature value; security light status; Intruder or no; etc

Figure 8. Typical conducted tests and results.

\section{Conclusion}

A fully functional system for security and control of a local area from several survey points was established; the system is cost-effective and remotely accessible. Using here RFbased sensor sub-nodes allows a survey of inaccessible locations and also extend the area under survey compare to the very limited area when only direct attached sensors to central processing unit are used. The sub-nodes therefore collect data from their respective survey points and communicate to the central processing unit which also stands as a main node and owner's remote access terminal of the system. This system can be installed in every home irrespective of societal class. In addition, nearly every local resident has a mobile and can easily manipulate it or at least can open an SMS and answer a call which makes the system very convenient and user-friendly. This system can also be implemented anywhere needing follow up of security and environmental indicators such as temperature, pollutant and pluviometry. Using GSM makes it low cost and remotely accessible especially with the SMS service it provides; it also provides a bidirectional communication ability.

The future scope of this project will include adding a security Camera to the system; that will allow a snapping of pictures as soon as an intruder is detected; those images can be stored in a SD card to be watched afterward.

\section{References}

[1] J. Teel, "Comparison of Wireless Technologies (Bluetooth, WiFi, BLE, Zigbee, Z-Wave, 6LowPAN, NFC, WiFi Direct, GSM, LTE, LoRa, NB-IoT, and LTE-M)," 7 November 2018. [Online]. Available: http://www.predictabledesigns.com. [Accessed 3 October 2019].

[2] O. G. Aju, "A Survey of ZigBee Wireless Sensor Network Technology: Topology, Applications and Challenges," International Journal of Computer Applications, vol. 130, no. 9, pp. 47-55, November 2015. 
[3] J. Armstrong and C. R. Helps, "Comparative evaluation of ZigBee and Bluetooth: Embedded wireless network technologies for students and Designers," American Society for Engineering Education, pp. 1-12, 2007.

[4] S. Akhter, "GSM-SMS technology for controlling home appliances remotely," International Journal of Computer Aided Engineering and Technology, pp. 1-5, January 2009.

[5] S. R. Khan, A. A. Mansur, A. Kabir, S. Jaman and N. Chowdhury, "Design and Implementation of Low Cost Home Security System Using GSM Network," International Journal of Scientific \& Engineering Research, vol. 3, no. 3, 2012.

[6] C. Bhadranna, "GSM Based Computer Lab Security System Using PIR Sensors," International Journal of Computer Science and Mobile Computing, vol. 6, no. 12, pp. 123-127, December 2017.

[7] Y. Sharif, A. Gupta, R. Patni, R. Sharma, S. Shrimali and S. S. Nirban, "GSM Based Home Automation System," International Journal of Electrical and Electronics Research, vol. 5, no. 2, pp. 1-4, April - June 2017.

[8] A. Sarkar, S. Sultana and M. H. Rahman, "A Smart Control System of Home Appliances Using SMS," Global Journal of Researches in Engineering: For Electrical and Electronics Engineering, vol. 17, no. 1, pp. 1-7, 2017.

[9] S. Chattoraj, "Smart Home Automation based on different sensors and Arduino as the master controller," International Journal of Scientific and Research Publications, vol. 5, no. 10, pp. 1-4, October 2015.

[10] Pierre Tsafack, Jean Kamdem, Bruno Allard. Wake-Up RF Communication Node Design and Use for Communal Living and Emergency Alert in Remote Areas of Developing Countries. Applied Engineering. Vol. 3, No. 1, 2019, pp. 65-70. doi: 10.11648/j.ae.20190301.18.
[11] D. K. Morgan, "Datasheet on CD4046B Phase-Locked Loop: A Versatile Building Block for Micropower Digital and Analog Applications," TEXAS INSTRUMENTS, 2003.

[12] A. M. Lonzetta, P. Cope, J. Campbell, B. J. Mohd and T. Hayajneh, "Security Vulnerabilities in Bluetooth Technology as used in IoT," Journal of Sensor and Actuator Networks, vol. 7, no. 28, July 2018.

[13] Rerkrai, K.; Jardak, C.; Kovacevic, A.; Riihijärvi, J.; Mähönen, P. Demo abstract: Survivable and scalableWSN solution for environmental monitoring in harsh conditions. In proceedings of 6th European conference on wireless sensor network, Cork, Ireland, U.K, february 11-13, 2009.

[14] Murty, R. N.; Mainland, G.; Rose, I.; Choudhury, A. R.; Gosain, A.; Bers, J.; Welsh, M. CitySense: An Urban-Scale Wireless Sensor Network and Testbed. In Proceedings of the 8th IEEE Conference on Technologies for Homeland Security, Waltham, MA, USA; IEEE Computer Society: Waltham, MA, USA, 2008

[15] Roderick Shepherd, Stephen Beirne, King Tong Lau, Brian Corcoran, Dermot Diamond, Monitoring chemical plumes in an environmental sensing chamber with a wireless chemical sensor network, Sensors and Actuators B: ChemicalVolume 121, Issue 1, Special Issue: 25th Anniversary of Sensors and Actuators B: Chemical, 30 January 2007.

[16] Anurag Kumar et al. Wireless Sensor Networks for Human Intruder Detection, Journal of the Indian Institute of Science VOL 90: 3 Jul-Sep 2010.

[17] Dipanjan Bhattacharjee et. al. Design and Development of Wireless Sensor Node/ (IJCSE) International Journal on Computer Science and Engineering. Vol. 02, No. 07, 2010, 2431-2438 